\title{
Modeling graphic subsystem for M5 simulator
}

\begin{abstract}
In computer architecture research and design, simulator is a viable alternative for researchers to evaluate the functionality and performance of the future computer systems architecture. The cost and timeline to complete a project tremendously can be reduced with simulators, as it allows a quick quantitative evaluation of the wide range of architectures in computer systems, such as CPU, memory and I/O system. The M5 simulator, is one of the open source computer architecture available. This paper propose a graphic subsystem for the existing M5 simulator to allow research and development activities in the area especially for Graphic Processing Unit (GPU). The GPU model chosen for the M5 simulator is the PCI based Voodoo 3 GPU, manufactured by the 3Dfx Interactive Inc. The functionality of the built GPU model are evaluated by using several software engineering testing methodologies, and the programmability of the model is tested by using the Glide API, the 3D graphics API developed for the Voodoo Graphics 3D accelerator cards.
\end{abstract}

Keyword: M5 simulator; Graphic subsystem; Voodoo 3 GPU 